\title{
Innovative Formula for Management of Dermatological Disorders Associated with Chediak Higashi
}

\author{
Manal MY1 and Nermin MY2* \\ ${ }^{1}$ Chemist and pharmacist, Egypt \\ ${ }^{2}$ Assistant lecturer-periodontology department, faculty of dentistry, MSA University, \\ Egypt
}

*Corresponding author: Nermin Mohammed Ahmed Yussif, Assistant lecturerperiodontology department, faculty of dentistry, MSA University, Egypt, Tel: +2/01118271929; E-mail: dr_nermin_yusuf@yahoo.com

\section{Abstract}

Chediak-Higashi syndrome is a rare autosomal recessive condition with variant stages of systemic manifestations. To date, the management of skin lesions depends mainly upon antibiotics and corticosteroids. The current study represents an innovative topical formulation depending upon natural agents.

Keywords: Chediak-Higashi syndrome; Vitamins; Dermal Lesions

\section{Introduction}

Chediak-Higashi syndrome is a rare familial disease that was firstly discovered in 1943. Up till now, nearly 500 patients where reported manifesting the disease worldwide. It mainly affects granulocytes as white blood cells (neutrophils, macrophages, lymphocytes and natural killer cells), melanocytes, neurons and platelets. The excretory functions and healing power of the cellular membrane are mainly affected with different degrees $[1,2]$.

On the histopathological level, intracellular giant cytoplasmic organelles could be detected in relation to the affected cells. Furthermore, aggregation, clumping and coalescence of the latter granules could be detectedcausing precipitations at the peripheral blood vessels or intracellularly $[1,2]$.

While, on the clinical level, the affected patients suffer from various manifestations. Higher mortality rate was reported between the affected patients. The disease usually reveals 3 stages; childhood, adolescent and adult forms. About 10-15\% of patients underwent childhood, adolescence and adulthood without accelerating phase. There are 2 different phenotypes that were reported in the literature. The first phenotype revealshigher levels of melanocytes dysfunction causing oculocutanenous albinism affecting skin, hair and eyes. While the second phenotype usually affects dark skinned (speckled hyperpigmentation) individuals. Albino phenotype is the most common, the easiest and the earliest to be diagnosed when compared with the other phenotype $[2,3]$.

Hemophagocytic lymphohistiocytosis (accelerated phase) is a fatal phase affecting $50-85 \%$ of chediak higashi patients causing their death. The accelerate phase manifested as fever, lymphadenopathy, enlargement and dysfunction of liver and spleen resulting in bleeding tendency. They exhibit mild stages of pigmentation, infectionsand neurological disorders as dementia, 


\section{Medical Journal of Clinical Trials \& Case Studies}

peripheral neuropathy, Parkinsonism, tremors and bleeding $[2,3]$.

Death usually occurs due to the recurrent infections, prolonged uncontrolled bleeding and lymphohistocytic infiltration of all organs [1].

Melanocytes are large dendritic cells which produce melanin and transfer melanosomes to the surrounding keratinocytes. They have several immunological and protecting functions $[1,3]$.

The white blood cells' dysfunction is the main etiology behind recurrent infections that occurred during the different phases of the disease. It is associated with impaired humoral and cellular immunity. It causes defective chemotaxis, killing, degranulation, cytotoxicity and phagocytosis of neutrophils, natural killer cells and monocytes. Deficient antibody production is also reported [1].

Infections could be bacterial, viral or fungal in origin which causes occulocutaneous, liver, spleen, renal and lung disorders. The affected patients are mainly suspected to staphylococcus aureus and beta hemolytic streptococcus [1].

The dermatological clinical picture is highly pathognomonic and diagnostic. It affects about $12.8 \%$ of the patients which could be secondly infected and become refractory to treatment. It is a combination of infection induced dermatitis (eczematous dermatitis), telangiectasia, erythroderma, crusted granulomatous plaques and hyperkeratosis (scales and patches). The recurrent dermal disorder regards to immune disorders and sun exposure. The infections are mainly bacterial (staphylococcus) or candidal (chronic localized candidiasis) or viral (AIDS). Nails, scalp and mucous membranes are also susceptible to infections [3].

Bleeding disorders are one of the main obstacles which need to be managed especially during surgical procedures. Bleeding regards to defective platelet intracellular granules and functions [1]. Petechial rashes were reported [4].

Up till now, chediak Higashi syndrome has no definite treatment. The management of the associated manifestations depend upon 3 stages; supportive therapy to control the acute condition and complications; controlling the acute phase (accelerated phase). The supportive therapy depends mainly on the early diagnosis of the disease, patient education, hygiene measures, meticulous dental care, skin protection using sun block, eye protection with sunglasses, vaccines (inactivated only), longer antimicrobial course (2-3 times longer than normal individuals), avoidance of drugs that causes temporary or permanent platelet dysfunction (aspirin, NSAIDs). During dental intervention, preference of minimally invasive techniques as subcutaneous route instead of intramuscular technique, usage of antihemorrhage agents as antifibrinolytic agents, desmopressin. Carbohydrate rich diet is mostly preferred. The patient has to be referred to dermatologist. The characteristic dermatological lesions are due to recurrent cutaneous infections resist treatment [5].

\section{Case report}

A 12 years old albino girl was admitted to the faculty of dentistry-Cairo University, suffering from Chediak Higashi syndrome which was previously diagnosed 5 years ago. The patient complained from advanced dental problems. During clinical examination, extensive dermal lesions related to palms and soles were detected. The patient was referred to a dermatologist in order to control the progression of the dermal lesions that last for several years ago.

A detailed questionnaire was performed in order to examine the severity of the disease. The lesions were described as elevated large tender pruritic nodules that cover the palms. The lesions become ulcerated and heal with scar tissue. The soles showed thick cornified layer. Unfortunately, the dermal lesions did not show any response to the systemic and local pharmacological agents which mainly depend upon antibiotics and corticosteroids. The acute attacks were mainly provoked during winter and upon exposure to sun.

Meticulous tailor made supportive treatment plan was performed in order to suit and modify her life style. The patient has to be away from any stressful conditions. Strict dietary recommendations were prescribed depending upon sugar, salt and wheat free diet to improve the function the impaired immune cells and reduce the GIT manifestations. Her diet depends mainly on rice, protein (milk, meat, fishes) and fibers (Fruits and vegetable). Daily vitamin $C$ and vitamin $D$ oral drops with minimal dosages were prescribed in order to improve the total performance of the patient. A topical dermal formulation was constructed via an expert chemist and pharmacist. The new formula depends mainly on providing a protecting coat to the underlying dermal lesions especially during the acute attacks, protection against sun, infection and other environmental conditions and finally, supplies the inflamed tissues with the needed essential agents important for healing. 


\section{Medical Journal of Clinical Trials \& Case Studies}

The formula composed mainly of a thick fatty base containing essential vitamins without preservatives. The patient was asked to use it in minute amounts daily before sleep. She was also instructed to clean the lesions carefully prior the application. were disappeared with great improvement of the skin texture. Total disappearance of skin purities was also reported (Figure 1). The patient was asked to continue the usage of this formula. Long term studies of the used formula were needed.

After 1 month, the patient was recalled for regular checkup and maintenance. Nearly $95 \%$ of the lesions

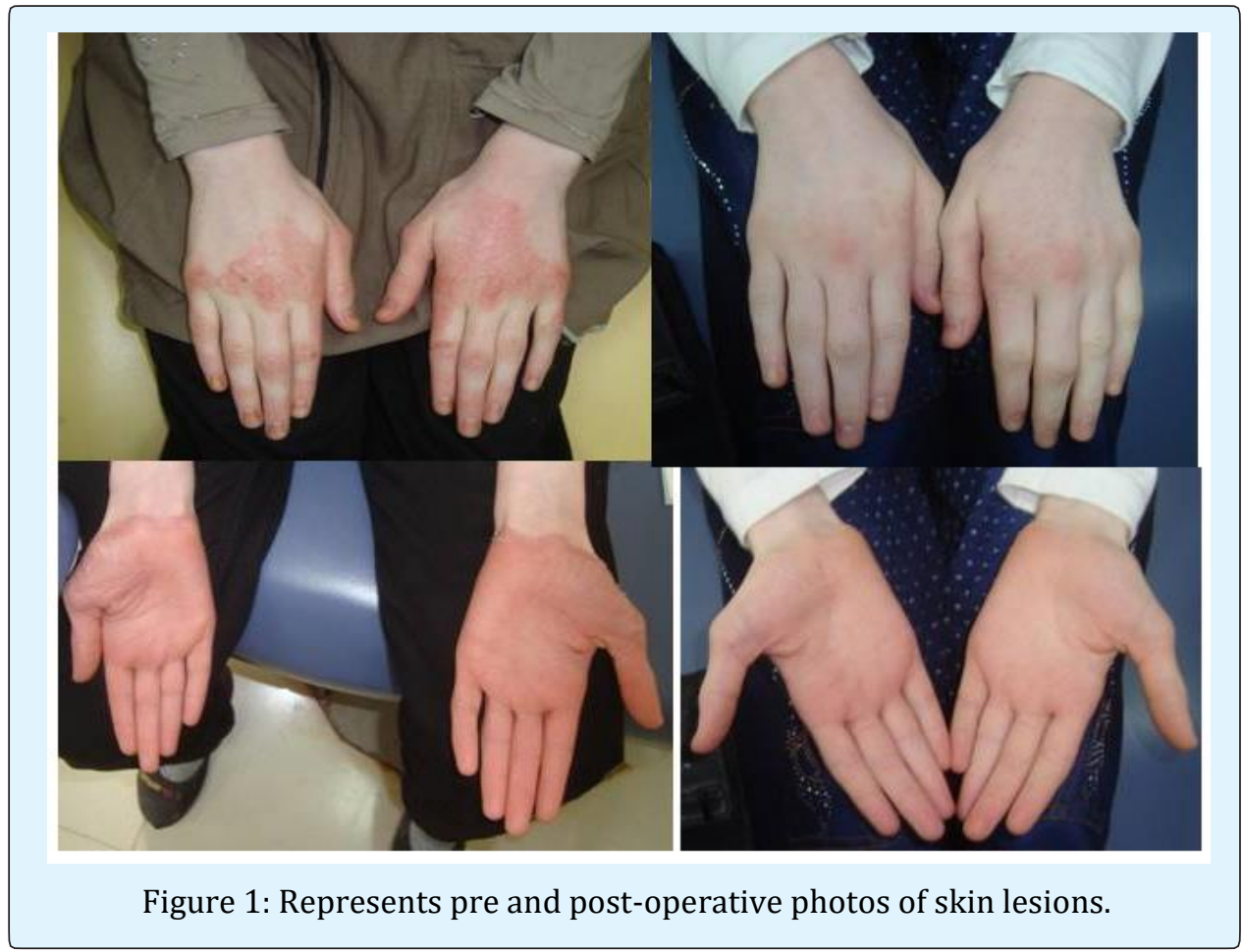

\section{Conclusion}

It was concluded that the new formula could provide a clinically significant improvement to the treated lesions of one of the most difficult and rare diseases that ever seen. The long-term usage of such formula could provide a great protection to the treated patient without the usage of corticosteroids or antibiotics.

\section{References}

1. Rezende KM, Canela AH, Ortega AO, Tintel C, Bonecker M (2013) Chediak-Higashi Syndrome and Premature Exfoliation of Primary Teeth. Brazilian Dental Journal 24(6): 667-670.

2. Lozano M, Rivera J, Sánchez-Guiu I, Vicente V (2014) Towards the targeted management of Chediak-
Higashi syndrome. Orphanet Journal of Rare Diseases 9: 132 .

3. Moin A, Farhoudi A, Moin M, Pourpak Z, Bazargan N (2006) Cutaneous Manifestations of Primary Immunodeficiency Diseases in Children. Iran J Allergy Asthma Immunol 5(3): 121-126.

4. Akbayram S, Akgun C, Basaranoglu M, Kaya A, Balta G, et al. A Case of Chediak-Higashi Syndrome Presented with Hemophagocytic Lymphohistiocytosis. International Journal of Hematology and Oncology 21.

5. Kirzioğlu Z, Altun AC (2008) Griscelli syndrome: A case report of Reye's syndrome and atopic dermatitis history. J Indian Soc Pedod Prevent Dent 26(3): S118S120.

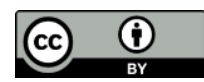

Copyright@ Manal MY and Nermin MY. 